\title{
In memoriam Marco Mumenthaler
}

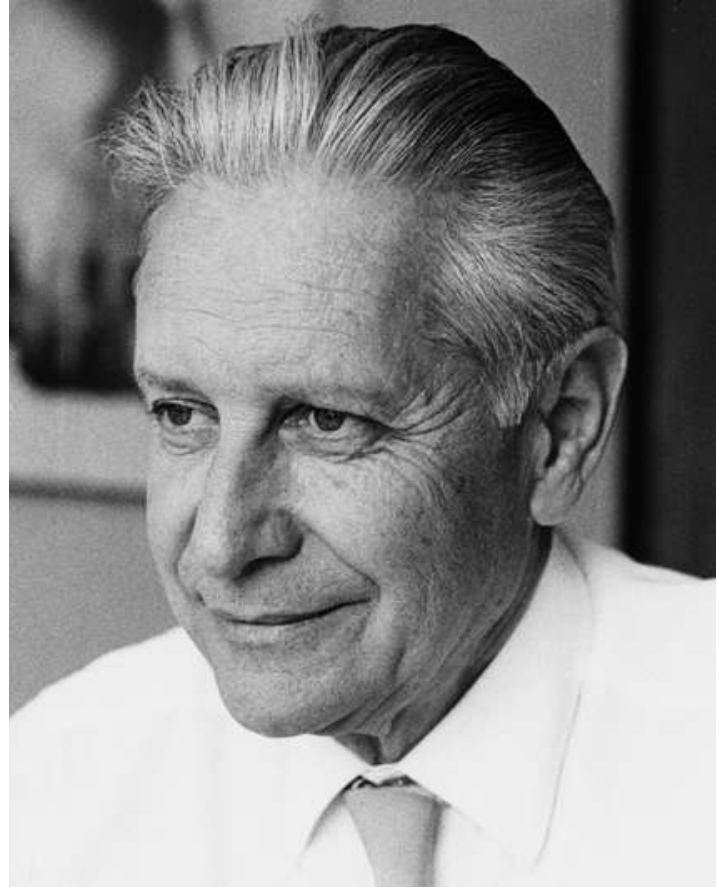

Prof. Dr. med.

Marco Mumenthaler 1925-2016

(Foto von 1989)
Am 30. Januar 2016 verstarb Marco Mumenthaler, ein hervorragender Arzt, klinischer Forscher und Lehrer. Er trug wesentlich zur Entwicklung der modernen Neurologie bei und prägte namhaft die Neurologie im deutschsprachigen Raum.

\section{Werdegang}

Marco Mumenthaler, geboren in Bern, wuchs als Sohn von Auslandschweizern in Varese und Mailand und auch bei seinen Grosseltern in der Leventina auf, absolvierte die Matura während des 2. Weltkrieges in Mailand, studierte Medizin in Zürich, Paris, Amsterdam und schloss sein Studium 1950 in Basel ab. Es folgte eine erste Assistentenstelle in Kardiologie in Paris und anschliessend Stellen in Psychiatrie, Neurochirurgie und Innerer Medizin. Eigentlich wollte er Psychiater werden, aber - vor allem motiviert durch seinen neurochirurgischen Lehrer Prof. Hugo Krayenbühl - wählte er die Neurologie und habilitierte sich an der Universität Zürich 1960 unter Prof. Fritz Lüthy. 1960/61 verbrachte er mit seiner Familie ein Jahr in den USA, wo er sich am National Institute of Health in Bethesda bei Prof. Milton Shy und Prof. King Engel der Erforschung der Muskelkrankheiten widmete. Kaum zurück in der Schweiz wurde durch den frühen Tod von
Rolf Magun die neurologische Chefarztstelle mit Extraordinariat in Bern frei, die er 1962 übernahm und ab 1966 als Ordinarius und Klinikdirektor bis zu seiner Emeritierung im Herbst 1990 innehatte. In diesen 28 Jahren baute er mit unermüdlicher Schaffenskraft die neurologische Abteilung des Inselspitals zu einer hervorragenden Universitätsklinik aus, sodass seine Klinik und er als Persönlichkeit nicht nur in der Schweiz, sondern auch weit über die Landesgrenzen hinweg einen exzellenten Ruf genossen. Ein Jahr nach seiner Emeritierung siedelte er nach Zürich um und arbeitete mit Hingabe als Facharzt für Neurologie weiter.

\section{Brillanter Lehrer und Redner}

Professor Mumenthaler engagierte sich während seiner ganzen klinischen Tätigkeit nicht nur für Patienten, sondern immer auch für Wissenschaft und Lehre. Als Klinikchef war er streng und präzise in der Sache und allen Mitarbeitern gegenüber stets gerecht und gütig. Messerscharfes analytisches Denken, Formulierungen in alemannischer Prägnanz und didaktisches Geschick machten ihn zu einem brillanten Lehrer und Redner bis ins hohe Alter. Legendär war seine Gabe, komplizierte medizinische oder juristische Sachverhalte - etwa im Rahmen von Gutachten - aus dem Stegreif in druckreifer Qualität ins Diktaphon zu sprechen. Selbst mit über 80 Jahren erntete er als Referent bei der Deutschen Gesellschaft für Neurologie stehenden Applaus.

\section{Zahlreiche Publikationen}

Prof. Mumenthalers Forschungsinteresse war ausgesprochen klinisch. Er veröffentlichte unzählige wissenschaftliche Arbeiten, zunächst vor allem über

\section{Selbst mit über 80 Jahren erntete er als} Referent bei der Deutschen Gesellschaft für Neurologie stehenden Applaus.

Muskelerkrankungen, später zu verschiedenen praktisch wichtigen Themen der Neurologie, und immer legte er Wert auf gute Didaktik. Zu den wissenschaftlichen Arbeiten kamen zahlreiche Monographien und Bücher hinzu. Erwähnt seien seine Habilitationsschrift über Ulnarisparesen, sein Lehrbuch der Neurologie (13 Auflagen), das Kurzlehrbuch der Neurologie (4 Auflagen), die Läsionen peripherer Nerven (10 Auf- 
lagen) und die Differentialdiagnose der Neurologie (10 Auflagen). Mit diesen und weiteren Büchern prägte er Generationen von Ärzten und insbesondere Neu-

\section{Sein Lehrbuch der Neurologie wurde in 14 Sprachen übersetzt.}

rologen. Seine Publikationen erhielten nicht nur im deutschsprachigen Raum Beachtung. Sein Lehrbuch der Neurologie wurde in 14 Sprachen übersetzt.

\section{Universitäres und politisches Engagement}

Neben der Tätigkeit als Chefarzt und Hochschullehrer engagierte sich Professor Mumenthaler auch in universitären und standespolitischen Belangen, wo ihm seine Überzeugungskraft und Eloquenz in drei Landesprachen und Englisch zustatten kamen. Während Jahren war er Sekretär der Medizinischen Fakultät, Präsident des Ärztekollegiums des Inselspitals, Präsident der Medizinisch-Ethischen Kommission der Universität Bern und in den Jahren 1989 bis 1991 während zweier Jahre Rektor der Universität Bern. In der schweizerischen ärztlichen Standesorganisation FMH war er langjähriger Vorsitzender der Weiterbildungskonferenz für Ärzte, während Jahren Vorsitzender des ärztlichen Beirates der Schweizerischen Gesellschaft für Muskelkrankheiten und von 1969 bis 1971 Präsident der Schweizerischen Neurologischen Gesellschaft. Militärisch bekleidete er als Major der Sanität die Funktion als Vorsitzender einer sanitarischen Untersuchungskommission und war mehrere Jahre Vorsitzender der Eidgenössischen Kommission für Kriegsmedizin. Von 1989 bis 1993 war er Mitglied des Internationalen Komitees vom Roten Kreuz in Genf und nahm an zahlreichen Missionen in Kriegs- und Krisengebieten teil, u.a. in Georgien, Afghanistan, Korea, Irak, Somalia, Äthiopien und Eritrea.

\section{Zahlreiche Ehrungen}

Das erfolgreiche und unermüdliche Schaffen brachte Prof. Mumenthaler zahlreiche Ehrungen und viele Mitgliedschaften in wissenschaftlichen Gesellschaften der Schweiz, Europas und Nordamerikas. Erwähnt sei hier lediglich das Ehrenpräsidium der Schweizerischen Neurologischen Gesellschaft.

Nach seinem 80. Geburtstag nahm die berufliche Betätigung allmählich ab. Geistig blieb Prof. Mumenthaler jedoch präsent und rege und vertiefte sich immer mehr auch in nicht-medizinische Literatur. Zunehmend

In der schweizerischen ärztlichen Standesorganisation FMH war er langjähriger Vorsitzender der Weiterbildungskonferenz für Ärzte.

schwächte ihn eine Herzinsuffizienz, so dass seine körperliche Leistungsfähigkeit und sein Bewegungsradius nach einer akuten Dekompensation im Herbst 2012 eingeschränkt blieben. Im vergangenen Dezember erlitt er zusätzlich eine Pneumonie, an deren Folgen er nun verstarb. Seinen Angehörigen entbieten wir unser herzliches Beileid. Marco Mumenthaler wird uns als prägende Persönlichkeit und lieber Freund in Erinnerung bleiben.

Heinrich Mattle, Christian W. Hess und Claudio Bassetti, Bern 